\title{
Standalone Inertial Pocket Navigation System
}

\author{
Estefania Munoz Diaz and Ana Luz Mendiguchia Gonzalez and Fabian de Ponte Müller \\ German Aerospace Center (DLR) \\ Institute of Communications and Navigation \\ Oberpfaffenhofen, 82234 Wessling, Germany \\ Email: Estefania.Munoz@dlr.de
}

\begin{abstract}
Positioning applications became more important in recent years not only for security applications, but also for the mass market. Having a pedestrian navigation system embedded in a mobile phone is a realistic solution since it is equipped with low-cost sensors and the smartphone is located in a nonobstructive way. The location of the smartphone is important, since the position estimation process depends on it. Therefore, we propose to distinguish between pocket or bag, phoning, texting and swinging. We present a standalone inertial pocket navigation system based on an inertial measurement unit. For the computation of the orientation, we have developed an attitude estimator based on an unscented Kalman filter. The update stage has two different updates based on the acceleration and the magnetic field. Therefore, a zero acceleration detector, a magnetic disturbances detector and a static periods detector have been developed. The odometry in our navigation system is computed through an extended Kalman filter. The position is predicted with a movement model which is periodically updated through position corrections computed by the position computer. It comprises a step detector and a step length estimator based on the norm of the acceleration. The performance of our attitude estimator in comparison with the ground truth orientation is shown. The rest of the handheld positions are also tested for orientation. Likewise, we show pocket odometries of different users with the floor plan superimposed.
\end{abstract}

Index Terms-Pocket, navigation, attitude estimator, magnetic field, odometry, heading, handheld.

\section{INTRODUCTION}

The market for positioning applications is rising in recent years for both, commercial and professional users. So far, most positioning applications are based on Global Navigation Satellite Systems (GNSSs), such as the American Global Positioning System (GPS) or the European Galileo. However, due to the lack of visible satellites, the availability of GNSS is degraded in certain scenarios such as urban canyons or indoors. The use of inertial measurement units (IMUs) is a viable solution to address this problem because they are able to provide human odometries [1] and show promising potential for reliable indoor pedestrian navigation. Additionally, IMUs are infrastructure independent.

However, micro electromechanical systems (MEMS) based IMUs have to face problems such as drifting sensors. Many authors [2], [3], [4], [5] use the rest phase of the foot to limit the growth of errors. Foxlin [3] was the first to propose an extended Kalman filter (EKF) to estimate and subtract the errors with the zero velocity updates (ZUPT) when the sensor does not move. There are more suitable solutions like the

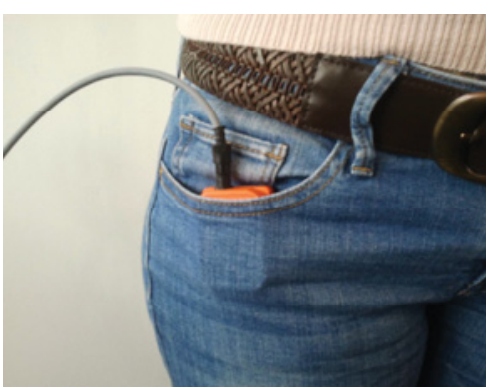

Fig. 1. The presented standalone inertial navigation system only requires introducing the IMU in the trousers' pocket and walking freely.

assumption of a fixed orientation during the rest phases. This is a zero angular rate update (ZARU) [2].

The previously explained methods help limiting the growth of errors, but they require having a foot-mounted IMU. Although this is an excellent solution for pedestrian indoor navigation, for the mass market it is more convenient to integrate the navigation system in the mobile phone. This is more practical and less obstructive than attaching an extra device to the shoe, however challenging since zero velocity corrections cannot be applied.

In this paper a standalone inertial pocket navigation system is presented. The system only requires introducing the IMU in the trousers' pocket and walking freely (Figure 1). The pocket does not have to be tight.

In Section II, a brief summary of the related work is gathered in order to offer an overview of the state of the art.

The developed inertial pocket navigation system is presented in Section III. Due to its complexity, we have dedicated a great part of this paper, the Section IV, to describe in detail the attitude estimator. In this section, the update equations system is carefully explained as well as the three developed detectors, i.e. the zero acceleration detector, the magnetic disturbances detector and the static periods detector. The position estimator is presented in Section V. The step detector and step length estimator are described in this section.

Finally, the experimental results are shown in Section VI. First, we show the performance of the attitude estimator compared to the ground truth given by a precise fiber optic gyroscope (FOG). We offer as well a brief attitude analysis of further handheld positions. Odometries of the described pocket navigation system are shown with the floor plan superimposed as a ground truth. 


\section{RELATED WORK}

There is a large amount of work in the area of pedestrian indoor localization. Respect to the attitude/heading determination for pedestrian dead-reckoning (PDR) approaches, only the heading or yaw angle, and not the complete attitude, seems to merit attention in the literature.

The combination of gyroscope and magnetic compass [6], [7] has widely been applied to obtain the heading for such a navigation systems. The integration of the gyroscope measurements provides it directly. Due to the biases, the long term drift in yaw needs to be corrected. The magnetometer, having been calibrated, is used with this purpose. The short term accuracy of the gyroscope helps detecting the short term external disturbances of the magnetometer. Especially in [8], [9], an interesting magnetic disturbance detector is developed.

There is also an initial prior work in the area of complete attitude determination [10]-[12]. Accelerometer, gyroscope and magnetometer measurements are combined to obtain it. Taking benefit of having two vectors, such as Earth magnetic field and gravity, whose directions and intensities are known, the orientation of the IMU can be extracted.

Bayesian approaches are commonly used to propagate dynamic variables such as Euler angles. In particular, the EKF has become the accepted basis for the major part of orientation algorithms [10], [12], due to the non-linearities of the equations used. However, a Madgwick filter, presented in [13], provides a non-bayesian based orientation algorithm. In this case, the gradient descent algorithm is used. A magnetic distortion compensation is also incorporated on it.

Respect to the sensor location, the previous work is mostly focused on foot-mounted techniques [14], [15]. With the footmounted IMU, strong corrections can be applied every step against the accumulated errors in strapdown systems.

For non-body-fixed sensor locations, PDR is the most suitable solution. In this case, the computation of the user's position uses

$$
x_{\text {step }_{i}}=x_{\text {step }_{i-1}}+l \cdot \cos (\psi)
$$

and

$$
y_{\text {step }_{i}}=y_{\text {step }_{i-1}}+l \cdot \sin (\psi),
$$

being $x_{\text {step }_{i}}$ the position in the $\mathrm{x}$-axis at the time stamp $i$, $y_{\text {step }_{i}}$ the position in the y-axis at the time stamp $i, l$ the estimated step length and $\psi$ the estimated heading.

For the step detection, there are different approaches depending on the IMU's location. The further the sensor is from the foot, the more difficult is to recognize a pattern. If the sensor is attached to the ankle [6], it is still possible to detect steps recognising patterns on the acceleration signal. However, for waist-/hip-mounted or pocket carried, this is not the most ingenious solution and three approaches are mainly used. The first one considers the peaks detection of the pelvis' vertical displacement [7], [16], [17]. The second method consists on detecting foot steps finding peaks in the variance of the vertical acceleration and introducing a threshold to avoid spurious step detections [18]. Thresholds are also used in the third approach, where the evaluated feature for the peaks detection is the norm of the complete acceleration.

Using the complete acceleration, this technique is independent of the sensor's orientation. The peaks correspond to the step occurrences because they are generated by the vertical impact when the foot hits the ground. Since the pattern of impact signal depends on the type of movement and it is greatly affected by the user's walking velocity, the determination of the thresholds for reliable step detection is challenging. Even considering this problem, distinguishing steps with the norm of the vertical or complete acceleration is also used when the sensors are handheld [19]-[22].

The main current techniques to estimate the step length can be classified depending on the IMU's location, as specified in [23], [24].

If the IMU is attached to the body near the center of mass:

- Based on a biomechanical model, where the kneeless biped is modeled as an inverted pendulum, and scaling the final estimation with a constant $K$ to calibrate for each user [16].

$$
l_{1}=K \cdot \sqrt{2 L h-h^{2}},
$$

where $l_{1}$ represents the estimated step length, $h$ the vertical displacement of the pelvis and $L$, the leg's length.

- Using an empirical relationship of the vertical acceleration and the step length [7], [19], [25].

$$
l_{2}=K \cdot \sqrt[4]{a_{\max _{v_{i}}}-a_{\min _{v_{i}}}},
$$

where $l_{2}$ represents the estimated step length, $K$ is a calibration value and $a_{\max _{v_{i}}}$ and $a_{\text {min }_{v_{i}}}$ are the maximum and minimum values of the vertical acceleration during step $i$.

If there are no restrictions on the IMU's placement [20], [21], [26]-[28], taking advantage of the relationship between step length $(l)$, height $(h)$, step frequency $\left(f_{\text {step }}\right)$ and some calibration parameters $(a, b, c)$ different for each user:

$$
l_{3}=h \cdot\left(a \cdot f_{\text {step }}+b\right)+c .
$$

\section{Inertial Pocket NAvigation System}

The inertial pocket navigation system will be described in this section. Firstly, we justify the choice of the filters and, lastly, we present the two main parts of it, i.e. the attitude estimator and the position estimator that will be described in Section IV and Section V respectively.

The inertial pocket navigation system should track the user's trajectory and process the data online as it arrives. For pedestrian navigation the interesting variable is the user's position. Bayesian approaches are the best option to estimate such dynamic variables. In this context, these variables are called states. For the dynamic state estimation this algorithm attempts to construct the posterior probability density function of the state based on all available information, including the received measurements. Every time a new measurement becomes available, it is sequentially filtered. Such a filter has two stages: prediction and update [29]. 


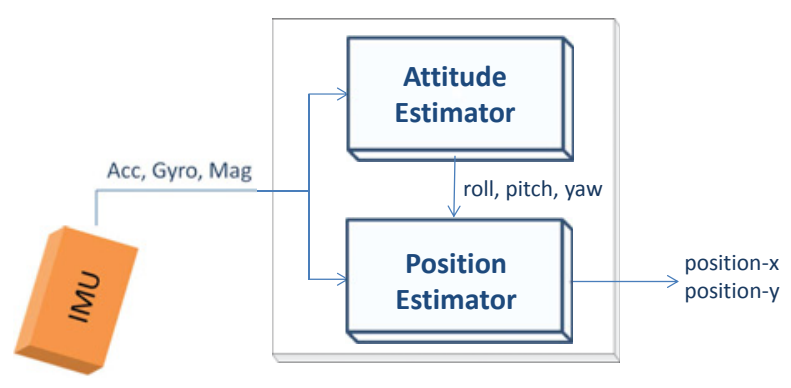

Fig. 2. Inertial pocket navigation system's block diagram. It has two modules, the attitude estimator and the position estimator, which are a UKF and a EKF respectively. The attitude estimator has 6 states which are the Euler angles and the gyroscope biases. The position estimator has 2 states which are the position- $\mathrm{X}$ and the position-y.

When certain constraints are hold, linearity and gaussianity, an optimal solution is tractable, which is a Kalman filter. There are also approximations to the optimal solution when these conditions are not fulfilled [29]. In this case, suboptimal algorithms have been used due to the non-linearities of the equations involved.

The unscented Kalman filter (UKF) propagates mean and covariance of the states through a linear regression between a set of points (sigma points) drawn from the prior distribution of the states. In the EKF the state distribution is propagated analytically through the first-order approximation of the Taylor series, thus the mean and covariance could be corrupted for high non-linearities.

The two main tasks of the inertial pocket navigation system are the estimation of the attitude and the estimation of the position. In Figure 2 a block diagram is shown.

For the attitude estimator filter, described in Section IV, a UKF has been chosen due to the high non-linearities of the equations involved. This filter has 6 states: the Euler angles, i.e. roll, pitch and yaw and the biases of the gyroscope. It counts with a prediction stage based on the integration of the gyroscope for the Euler angles and on an random walk model for the biases. For the update stage, corrections using acceleration and magnetic field are carried out. In order to detect periods of zero acceleration of the user, a detector has been developed. The magnetic field measurements are known to be distorted in certain scenarios, thus we have a magnetic disturbances detector. We have also implemented a static periods detector.

For the position estimator, described in Section V, a EKF has been chosen. The states are two: position-x and position$\mathrm{y}$. In the prediction stage the two dimensional position is estimated through a movement model. For the position update it has been developed a step detector and a step length estimator. The update occurs once per user's step. The needed heading is taken from the attitude estimator filter.

We have decided to divide the pocket navigation system in two parts because we want a modular architecture. This will allow us modifying separately the position estimator and the attitude estimator. The modular architecture allows also adding

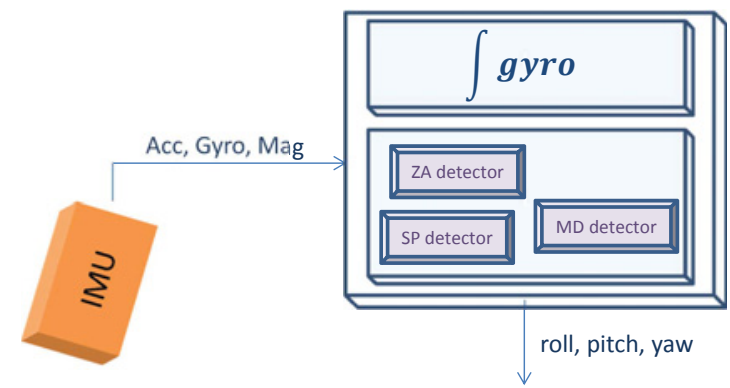

Fig. 3. Attitude estimator UKF filter. The light blue boxes represent the two stages, prediction and update. In this filter there are three detectors implemented to help applying the updates, the zero acceleration detector, the magnetic disturbances detector and the static periods detector.

other blocks to help navigation.

\section{Attitude Estimator}

In this section we describe the attitude estimator. All components such as the zero acceleration detector, the magnetic disturbances detector and the static periods detector are detailed. The structure of the prediction and update stage is carefully explained.

The attitude estimator is a UKF whose states are the Euler angles roll, pitch and yaw and the gyroscope biases.

As Figure 3 shows, the attitude estimator receives directly the accelerometer, gyroscope and magnetic field sensor measurements. The upper box in light blue represents the prediction stage. The prediction stage for the Euler angles consists of a simple integration of the turn rates. For clarity, only this part has been represented in the block diagram. The gyroscope's biases are predicted using a random walk model.

The lower light blue box represents the update stage. For applying the updates three specific detectors are needed.

During the periods of no-user-acceleration only the gravity acceleration is measured. Therefore, the zero acceleration assumption (ZAA) can be only applied few milliseconds every step and obviously when the user does not move. In order to correctly apply this, these no-acceleration periods have to be accurately detected. However, applying this update is not enough because the heading remains unobservable. The accelerometer only allows correcting roll and pitch.

The magnetic field intensity can be used for correcting the attitude. Magnetometer measurements are always available, however, in indoor environments and other challenging outdoor scenarios such as the vicinity of ferromagnetic materials, the magnetic field is distorted. It is not convenient to use directly the magnetometer measurements in these cases. In this work, we use magnetic corrections depending on the perturbation of the measured magnetic field. Therefore, it is necessary to identify when the magnetic field is distorted. The magnetic disturbances detector is based on both, changes on the magnetic intensity and changes on the orientation of the measured magnetic field.

The static periods detector gives an output when the user is static, i.e. standing, sitting or lying among others. During these 
periods, a realignment is done and the biases of the gyroscope are updated.

\section{A. Zero Acceleration Detector}

The zero acceleration detector is needed in this work for different applications.

This detector finds locally periods of zero or close to zero acceleration. Firstly, for simplicity, consider the case where the user is standing. For simplicity also consider free biases accelerometers. If no biases are present, the measured acceleration is only the gravity. The knowledge of the gravity vector offers information of the orientation of the IMU, specifically the roll and pitch. The orientation based on the gravity vector is heading independent.

Zero acceleration implies constant velocity. Obviously, if the user is standing the velocity is constant and equal to zero. Additionally, during the walk, periods of zero acceleration can be also found. The pedestrian's step has a pattern, acceleration and deceleration. Between those rely periods of zero acceleration. Since the sensor is placed in the pocket, no zero velocity can be found during the walk like using a foot-mounted sensor, but zero acceleration.

The formula used to find zero acceleration periods is

$$
\sqrt{\text { accel }_{X_{k}}^{2}+\text { accel }_{Y_{k}}^{2}+\text { accel }_{Z_{k}}^{2}}<\gamma,
$$

where accel $_{i_{k}}$ being $i=X, Y, Z$ is the acceleration of the $i$ axis measured on the time stamp $k$. The parameter $\gamma$ is defined based on the observation of the accelerometers to make the zero acceleration detector be active only when the left part of the inequation (6) is close to the gravity value.

\section{B. Magnetic Disturbances Detector}

The Earth's magnetic field is modeled as a dipole. At any location, it can be represented by a three-dimensional vector. For centuries, the Earth's magnetic field has been used for navigation purposes. The goal is to determine the direction of the magnetic North.

The angle between the magnetic and the true North is called declination. Facing the magnetic North, the angle the field makes with the horizon is called inclination. The Munich Earth Observatory has reported an average intensity of $48219.7 \mathrm{nT}$, an inclination angle of 64.23 degrees and a declination angle of 2.57 degrees for January 2014 [30]. These measurements have been taken in Fürstenfeldbruck, $15 \mathrm{~km}$ away from DLR.

The magnetometer measurements contain the information of the orientation of the sensor within the Earth's magnetic field. The heading is extracted as follows

$$
\psi=\tan \left(\frac{-m a g_{X}}{m a g_{Y}}\right)^{-1} \pm D,
$$

where $\psi$ is the heading measured in radians and $\operatorname{mag}_{i}$ where $i=X, Y$ is the magnetic field intensity for the $i$-axis. $D$ represents the declination angle.

Before using the magnetometer, it is recommendable to calibrate it [31]. The sensor was manually moved describing

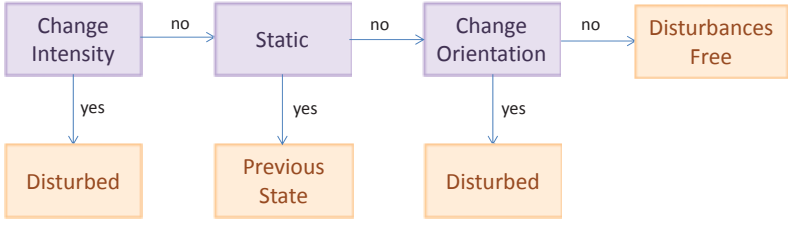

Fig. 4. Decision tree of the magnetic disturbances detector. If no changes on the intensity are detected, may be because the user is static. Perturbations may be present, however the magnetic field is short-term time-invariant. In this case there is no information to output a new state. If the user is not static the orientation is checked.

random paths in all directions and the raw data was recorded. A sphere is fitted through least-squares out from an ellipsoid made of uncalibrated data. The biases of all 3 magnetometer axis are set as the coordinates of the center of the sphere. The radius of the sphere is used to normalize the sensor measurements to the previously mentioned local magnetic field intensity. The calibration took place once in an outdoor field disturbances free.

However, as previously mentioned, the magnetometer measurements will be distorted due to the proximity of ferromagnetic materials and electric currents [32]. That means that, even if the magnetometer is properly calibrated, the measurements may be distorted if the user walks, for example, through the sidewalk close to parked cars.

Therefore, we have implemented a magnetic disturbances detector. It is based on two parameters: the intensity and the orientation of the magnetic field [33].

We have developed a magnetic disturbances detector based on the identification of the periods where the measured magnetic field is constant in intensity and orientation. The fact that the magnetic field is constant does not imply that it is disturbances free. We have identified two cases where the magnetic field is constant and although perturbed. The first occurs if the sensor is static. The user may be in a building where the magnetic field is completely distorted, however, if the user does not move, the distortion is constant in time. This false detection of a disturbances free magnetic field is avoided through the static periods detector. The other false detection arises when hard iron biases are measured by the sensor. The hard iron errors are caused by a magnetic source which causes a permanent field in all orientations. This is avoided in our case through a previous magnetometer calibration. Therefore, since these two situations are discarded, we consider our detector to be a magnetic disturbances detector.

Figure 4 represents the decision tree of the magnetic disturbances detector. Firstly, the changes on the intensity of the magnetic field are checked through the standard deviation and compared with the standard deviation of a non-disturbed magnetic field. The magnetic field intensity and accordingly its standard deviation have great variations with the high of the sensor respect to the floor. Figure 5 shows the intensity of a foot-mounted sensor compared with the intensity registered by a sensor in the pocket. The walk was recorded with both IMUs at the same time. The first part of the walk the user 


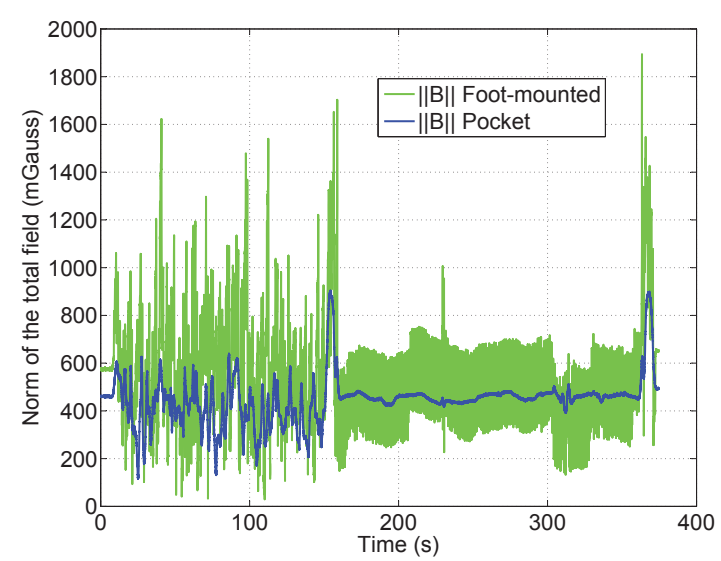

Fig. 5. The green curve represents the norm of the total magnetic field for a foot-mounted sensor and the blue curve represents the norm of the total magnetic field for the same walk with the sensor in the pocket. The first part of the walk was indoors, from the second 150 to the 360 the walk was outdoors and at the end the user enters again the building.

is indoors, then it goes outdoors and finally indoors again to the starting point. The difference on the starting value of both curves can be explained because, even if both IMUs are on the same user, the difference on the high causes much different perturbations. Around the second 150 the user goes outdoors and walks surrounding our office building. From $160-350$ seconds the user walks close to the parking and around the second 310 the user walks only $1 \mathrm{~m}$ away from a parked car.

If there is a change on the intensity, the magnetic field is disturbed. If not, may be the case that the user is static. We detect this through the static periods detector. The magnetic field in indoor environments is known to be constant in time and varying in direction and intensity. Magnetic signatures are stable over a period of several months [34] and strongly modulated in space [32]. Therefore, if the user is static (standing, lying, sitting...) we keep the previous state, disturbances free or disturbed. The initial hypothesis is a perturbed magnetic field.

If the user is not static, the change in orientation is checked. Obviously a change in orientation of the magnetic field could be due to a change in orientation of the user. Therefore, we verify through the gyroscope the change of the user's heading. If the user has rotated less than the change registered by the magnetometers, a perturbation of the magnetic field is present. We compute the magnetometer's change in orientation through the Equation (7) for the actual time stamp minus the same equation for the previous time stamp.

After the work realized for this magnetic disturbances detector we realized that the magnetic signatures are different at different heights for the same $(\mathrm{x}, \mathrm{y})$ position. Therefore, they are not only varying in $\mathrm{x}$ - and $\mathrm{y}$-axis, but in $\mathrm{z}$-axis.

\section{Static Periods Detector}

The static periods detector consists on sliding window buffers that control acceleration and gyroscope signals constantly. Through the Allan variance, the standard deviation of the white noise of both, accelerometers and gyroscopes, is known. Therefore, if the user is static, the mean of all accelerometer and gyroscope buffers should not be greater than this value.

The static periods detector gives its first output at the beginning of every walk, because the user is standing a couple of seconds. This is called the alignment phase. The zero acceleration principle is used for this phase. It is possible during the alignment phase to compute the biases of the gyroscope, because the sensor does not rotate. That means the sensor measures only the biases of the gyroscopes. On the contrary, it is not possible during the alignment phase to compute the biases of the accelerometers, because the knowledge of the gravity vector is not enough to distinguish roll and pitch from biases of $\mathrm{x}$ - and $\mathrm{y}$-axis. Therefore, during the alignment phase, we assume the accelerometers are biases free and everything the accelerometer measures is due to an initial rotation of the sensor. Thus, the biases of the accelerometer remain unobservable.

This alignment procedure is repeated every time the user is standing, not only at the beginning, but during the walk when they are identified by this detector. We take benefit of these periods for the realignment of the IMU, because it may move in the pocket during the walk. Additionally during these periods, we update the gyroscope's biases.

We use also this detector to help the magnetic disturbances detector, as explained in the previous subsection.

\section{Zero Acceleration Assumption (ZAA) Update}

The ZAA is an update for the Euler angles roll and pitch. As previously explained, with the knowledge of the gravity vector the yaw remains unobservable. The update equations are

$$
\phi=\tan \left(\frac{a c c_{Y}^{b}}{a c c_{Z}^{b}}\right)^{-1}
$$

and

$$
\theta=\tan \left(\frac{-a c c_{X}^{b}}{\sqrt{\left(a c c_{Y}^{b}\right)^{2}+\left(a c c_{Z}^{b}\right)^{2}}}\right)^{-1}
$$

where $\phi$ represents the roll angle, $\theta$ represents the pitch angle and $a c c_{i}^{b}$ for $i=X, Y, Z$ represents the acceleration measurement for the $i$-axis in the body frame.

Figure 6 shows the detected periods of zero acceleration for a 25 seconds walk. For a normal speed walk, that means not running, theoretically a zero acceleration interval will be every step detected. While standing, the zero acceleration is active the whole time.

\section{E. Magnetic Field Update}

The static periods detector and the magnetic disturbances detector help activating the magnetic field updates. Figure 7 shows the detected disturbances free periods during the same walk previously described in the Subsection IV-B.

Figure 7 shows that when the user is indoors the magnetic field is completely distorted. The two clear peaks pointing upwards were detected when the user goes through an automatic 


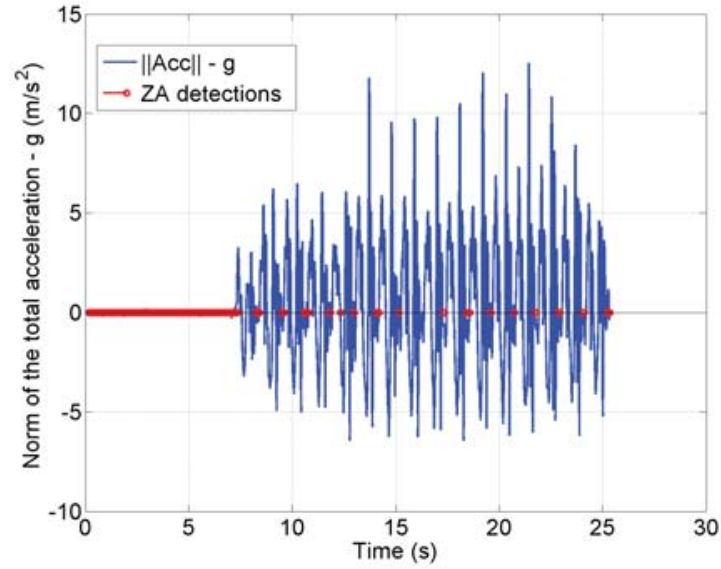

Fig. 6. The blue curve represents the norm of the total acceleration minus the local gravity value, measured during a walk. The first 7 seconds the user is standing. The red circles represent the periods where the detector identifies zero acceleration.

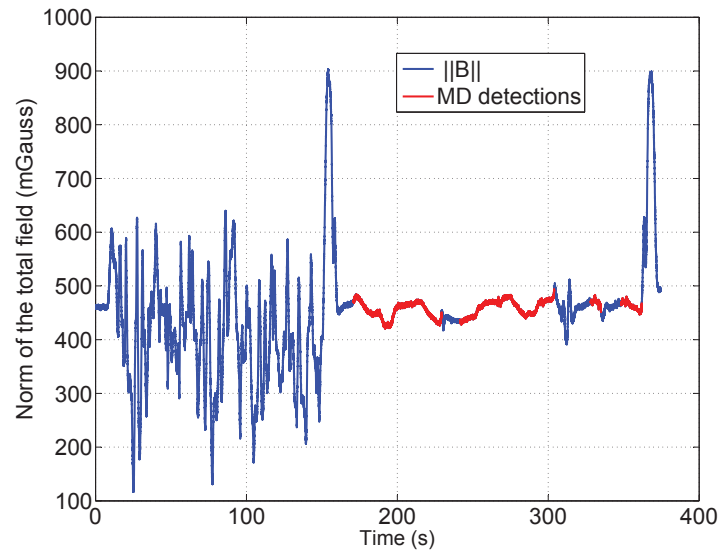

Fig. 7. The blue curve represents the norm of the total magnetic field for a sensor placed in the pocket. The red line shows the detections of the disturbances free magnetic field. The first part of the walk was indoors, from the second 150 to the 360 the walk was outdoors and at the end the user enters again the building. This is the same walk represented in Figure 5.

door, once for going outdoors and the last one for going into the building again. As explained before, even though the user was outdoors, the detected magnetic field is not completely clean because the user was walking close to the parking and approximately on the second 300 the user walks very close to a parked car. This creates a perturbation on the detected magnetic field.

If the magnetic field is disturbed, no direct computation of the yaw through Equation (7) can be used. However, when the user is outdoors and the measured magnetic field is disturbances free, this equation can be used to update the yaw.

For applying this update, it is necessary to project the magnetometer measurements from the body frame to the horizontal plane, being the rotation matrix

$\operatorname{mag}^{h}=\left[\begin{array}{ccc}\cos (\theta) & \sin (\theta) \sin (\phi) & \sin (\theta) \cos (\phi) \\ 0 & \cos (\phi) & -\sin (\phi) \\ -\sin (\theta) & \cos (\theta) \sin (\phi) & \cos (\theta) \cos (\phi)\end{array}\right] \overrightarrow{m a} g^{b}$,

where $m \vec{a} g^{b}$ and $m \vec{a} g^{h}$ are the magnetic measurements on the body frame and projected to the horizontal plane respectively. The roll angle is represented by $\phi$ and $\theta$ represents the pitch angle.

Once the magnetic measurements are projected onto the horizontal plane, Equation (7) can be used. This update presents good results for long-term corrections, however it requires projecting the magnetic measurements using the angles roll and pitch. Although the magnetic measurements are supposed to be error free because no disturbances are present, roll and pitch angles may contain errors which are introduced in the update of the yaw through the projection. Another source of errors is an incorrect magnetometer calibration.

However, the magnetic field has more information apart from the heading [8]. The errors in roll, pitch and yaw can be found through the magnetic field measurements, but only when the magnetic field is not perturbed. Therefore, the magnetic disturbances detector will be also used for enabling or disabling this update.

A disturbances free magnetic field implies that the measured magnetic field does not contain errors. Given the magnetic field at the time stamps $k-1$ and $k$, it is possible to find the rotation between both vectors due to the user motion as follows

$$
m \overrightarrow{a g} g_{k}^{b}=\vec{\omega}^{b} \times m \overrightarrow{a g_{k-1}^{b}},
$$

where $m a \vec{g}_{k-1}^{b}$ and $m \vec{a} g_{k}^{b}$ is the magnetic field vector in the body frame at time stamps $k-1$ and $k$ and $\vec{\omega}^{b}$ represents the rotation between these two vectors in the body frame.

As the magnetic field has no errors because it is not perturbed, we consider the resulting angles of the rotation, i.e. $\Delta \phi_{\text {mag }}, \Delta \theta_{\text {mag }}$ and $\Delta \psi_{\text {mag }}$, the error free rotation due to the user motion between the time stamp $k-1$ and $k$.

On the other hand, the rotation due to the user motion between the time stamp $k-1$ and $k$ can be also extracted by integrating the turn rates of the gyroscopes to get the Euler angles for both time stamps. Therefore,

$$
\begin{aligned}
& \Delta \phi_{g y r}=\phi_{k}-\phi_{k-1}, \\
& \Delta \theta_{g y r}=\theta_{k}-\theta_{k-1}, \\
& \Delta \psi_{g y r}=\psi_{k}-\psi_{k-1},
\end{aligned}
$$

being $\phi, \theta, \psi$ the Euler angles roll, pitch, yaw. However, this rotation contains errors due to the integration of the biases.

The idea is to use the rotation of the magnetic vector between the time stamp $k-1$ and $k$, which is error free, to correct the difference of the states roll, pitch, yaw between the time stamp $k-1$ and $k$, which contains errors due to the integration of the biases. 


\section{Position Estimator}

In this section we describe the position estimator and more in detail the structure of the prediction and update stages. The position estimator is a EKF whose states are the 2-dimensional position, i.e. position-x and position-y.

The aim of this filter is to sequentially compute the user's position. Therefore, the position estimator needs the estimation of the heading, which is sequentially provided by the attitude estimator.

In order to compute the user's position, this filter uses a movement model for the prediction of the position. For the position updates and the velocity computation we have developed a step detector and a step length estimator.

\section{A. Prediction Stage}

The prediction stage of the position estimator filter consists, as previously mentioned, on a movement model for the position. We have chosen the following human walk model

$$
\begin{aligned}
& \operatorname{pos} X_{k}=\operatorname{pos} X_{k-1}+\int_{k-1}^{k} \mathrm{vel} \cdot \cos \left(\psi_{k}\right), \\
& \operatorname{pos} Y_{k}=\operatorname{pos} Y_{k-1}+\int_{k-1}^{k} \mathrm{vel} \cdot \sin \left(\psi_{k}\right),
\end{aligned}
$$

where $\operatorname{pos} X$ and $\operatorname{pos} Y$ are the position estimates for the time stamps $k-1$ and $k$, vel is the mean velocity of the user and $\psi_{k}$ is the estimated heading angle for the time stamp $k$.

The last position is always available because it is a state, however, this movement model needs as well two external parameters such as the mean velocity of the user and the heading angle. As explained before, the heading angle is every time stamp provided by the attitude estimator. The mean velocity of the user is computed in the step detector and step length estimator.

\section{B. Update Stage}

Since the prediction human walk model needs the velocity of the user, a step detector and a step length estimator have been developed. The step length estimator provides as well position updates every step.

1) Step Detector: The step detector is based on the detection of consecutive minimum-maximum of the low-pass filtered norm of the acceleration. We have decided to use the norm of the acceleration instead of its vertical component, which is usually used in the literature, because the norm of the acceleration yields a detection independent of the IMU orientation.

In order to avoid false detections, some thresholds for the amplitude and the time between detections have to be set. The correct choice of these thresholds is one of the main difficulties of this technique. Therefore, we have decided to low-pass filter the acceleration. The filter smoothes the signal for its postprocessing as Figure 8 shows. In this figure, the green sticks represent the detected user steps. In our work we define a user step as the time between hits of the floor with every foot.

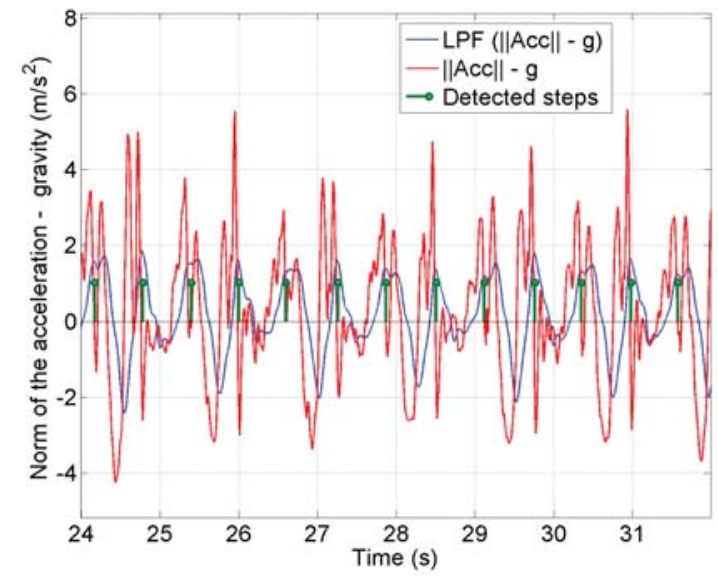

Fig. 8. The red curve represents the norm of the acceleration minus the local gravity value for a short period of a walk. The blue curve represents the same signal, but filtered for the same period of the walk. The green sticks represent the detected user steps.

2) Step Length Estimator: For the step length we have decided to use the empirical relationship between the maximum and minimum of the acceleration for each step, as Equation (4) shows. The parameter $K$ has to be adjusted due to the low-pass filter of the norm of the acceleration.

The combination of the step length and the heading gives a new position every user step which is used to update the prediction model.

\section{EXPERIMENTAL RESUlTS}

In order to assess the performance of the described inertial pocket navigation system, we have realized a set of experiments. In the first part of this section we focus on the attitude estimator. We have used a FOG as a ground truth. Then, we have decided to test our attitude estimator created for pocket located IMUs, with the rest of the handheld positions, i.e. swinging, calling and texting. These results are also shown with the FOG as a ground truth. Lastly, we show the performance of the complete inertial pocket navigation system with a odometry. As ground truth we plot the floor plan superimposed.

Two medium range IMUs, the MTx and MTw from Xsens, have been chosen. These devices have a 3 -axis accelerometer, a 3 -axis gyroscope and a 3 -axis magnetometer inside. The IMU is introduced in the pocket without a predefined orientation before starting the walk. The navigation system has been tested by 8 different users with different type of trousers and no restriction about the tightness of the trousers has been found.

\section{A. Attitude Estimator Experiments}

Firstly, we want to test the performance of the described attitude estimator. We have prepared a set of indoors-outdoors walks with different volunteers.

We have chosen an outdoor walk to assess the performance of the different update functions we have previously defined. Figure 9 shows the attitude angles roll and pitch for an 

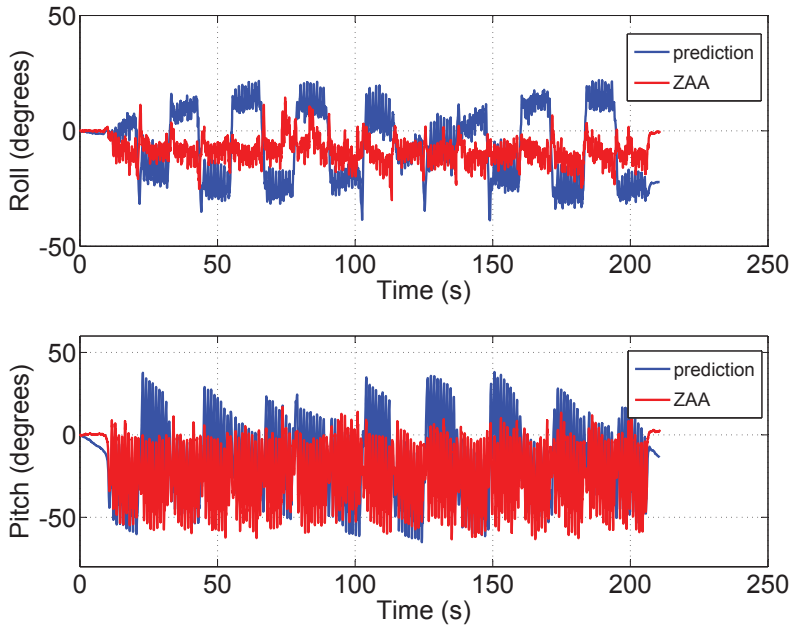

Fig. 9. The blue curves represent the roll and pitch angles of an outdoors walk processed with the attitude estimator without applying any update, only with the prediction stage. The red curves represent roll and pitch angles of the same walk process with the attitude estimator applying the ZAA update.

outdoors walk processed only with the prediction stage and with the ZAA update. It can be clearly seen the improvement in roll and pitch for the red curves, which have been processed with the ZAA update. The blue curves represent the same walk processed only with the prediction stage, without applying any update.

Figure 10 shows the heading angle for the same outdoor walk processed with and without the magnetic updates. This walk describes a round trip path with 16 turns of 180 degrees that can be clearly distinguished in the figure. The final value should be 2880 degrees, which is the sum of all turns. The blue curve, which was processed only with the prediction stage, reaches a final value of 2618 degrees. The green curve has been processed only with the yaw update of Equation (7) and it reaches a value of 2846 degrees. Finally, the red curve ends up with 2890 degrees. This figure shows the benefit of applying both magnetic updates.

Since it is not straight forward to analyze the difference between the green (only applying the yaw update of Equation( 7)) and the red curve (applying both magnetic updates) of Figure 10, we have decided to plot their odometries. Figure 11 shows the great impact of the heading for the odometries. The first subplot shows the true trajectory, a round trip path with 6 turns of 180 degrees with a length of 3.5 meters. The second subplot shows the odometries computed with the different headings of Figure 10. As previously explained, the blue curve for the heading accumulates more than 200 degrees of drift in a walk of approximately 200 seconds. The difference of the green and red heading curves can be more easily evaluated with their odometry curves. The red one keeps better the true trajectory path.

With Figure 11 we pretend to show the great impact of the heading in the odometry. This is not our final odometry result because, for generating these curves, only the magnetic updates were active. However, not all the errors the odometry

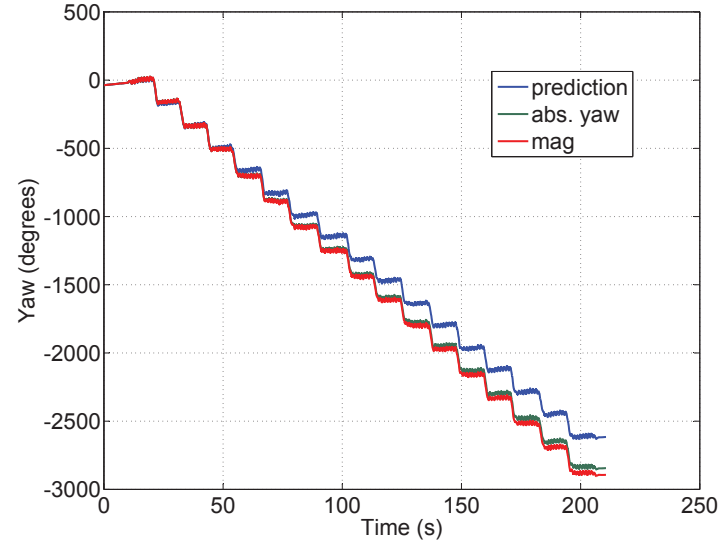

Fig. 10. The blue curve represents the yaw angle for the same outdoors walk as Figure 9 processed without applying any update, only with the prediction stage. The green curve has been computed applying only the absolute yaw update of Equation (7) and the red curve was computed applying both magnetic updates.

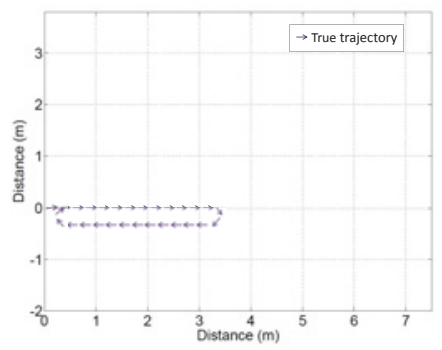

(a) True trajectory

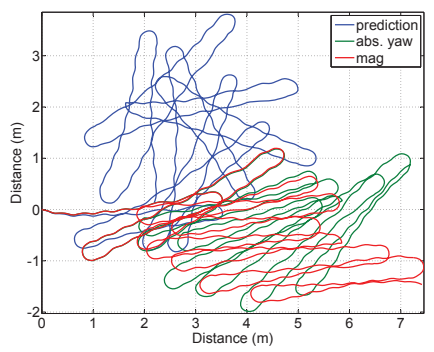

(b) Different update functions
Fig. 11. Odometries computed with the different headings of Figure 10.

shows have their origin in the attitude or heading estimation. This will be explained in the Subsection VI-A1.

Once the different update functions have been separately tested, the goal is to test the performance of the complete pocket attitude estimator in comparison with a ground truth. We have chosen the DSP-1750 FOG from KVH. Before considering this IMU as a ground truth, we have recorded 10 hours of data in the basement of our office building for analyzing its Allan variance. The FOG IMU is two orders of magnitude less noisy than our MEMS IMU and the biases noise is as well more than two orders of magnitude smaller.

The FOG is too large to introduce it in the pocket, as we always do with the MEMS IMU. Therefore, it has been attached with 4 screws to a solid wood base. The piece of wood is at the same time attached to the leg as Figure 12 shows. The optical IMU needs an external power supply. We have introduced the battery with the rest of hardware necessary for it to record the data in a metallic box. The MEMS IMU for all the experiments is attached with tape to the flat face of the FOG situated on the front. Due to the metallic box, the screws and the rest of the required hardware, it is convenient to recalibrate the MEMS IMU.

In Figures 13, 14 and 15, the Euler angles roll, pitch and yaw have been represented for an indoors walk. During the 


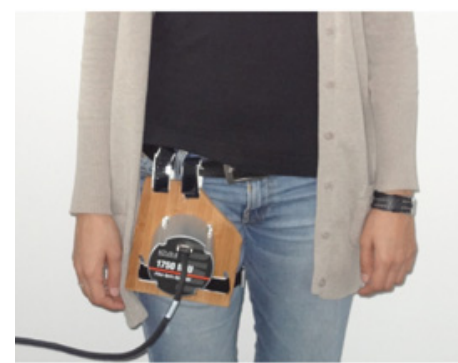

Fig. 12. This figure shows the solution we have found to attach the FOG to our leg at the high of the pocket because it is too large to introduce it directly in the pocket as we always do for the MEMS IMU.

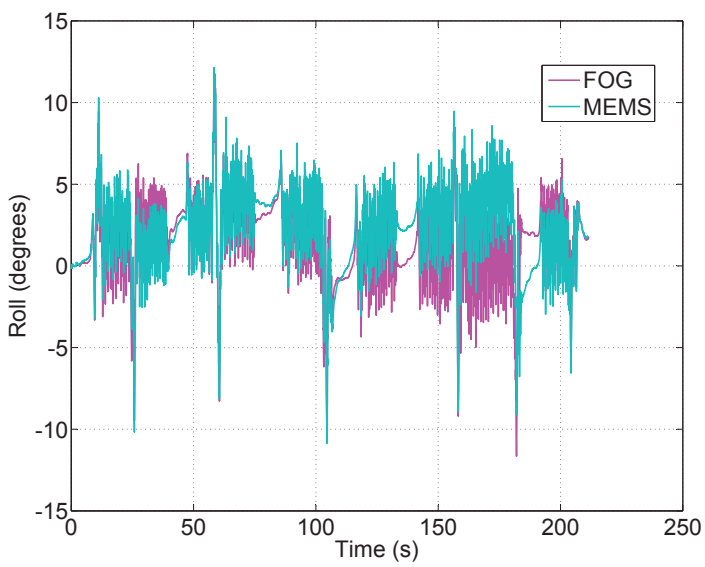

Fig. 13. The magenta curve represents the roll angle for an indoors walk computed with a FOG IMU which we consider as a ground truth. The turquoise curve represents the roll angle for the same walk processed with the pocket attitude estimator.

walk the volunteer was asked to do 5 short stops, which can be clearly seen in all angles as a straight line. The magenta curves represent for every case the ground truth obtained with the FOG IMU and the turquoise curves represent the same walk processed by our complete attitude estimator. For the processing of the FOG data, we have projected the raw turn rates from the sensor frame to the body frame and then we have applied a single integration. No correction of the biases or updates have been applied.

Figure 13 shows the roll angle for both IMUs and a clear displacement of the turquoise curve with respect to the magenta curve, which corresponds to the FOG IMU, can be seen. It is noticeable that, during the short stops where the user was asked to remain standing, the turquoise curve approximates to the magenta reference curve due to the ZAA updates. It is under investigation how to improve the roll angle results. The pitch angle represented in Figure 14 shows much better results. On the other hand, Figure 15 shows how the turquoise curve, which is computed with the pocket attitude estimator, starts slowly drifting almost since the beginning, reaching 18 degrees after 200 seconds. During the walk it can be clearly seen 3 turns of 180 degrees, 2 single 90 degrees turn at the beginning and at the end of the walk and 2 double 90 degrees turn in the second 60 and 160 . Even though we

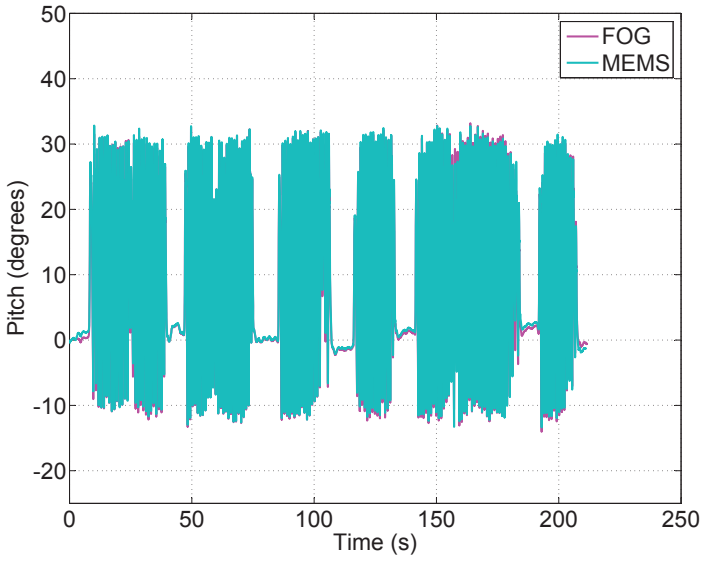

Fig. 14. The magenta curve represents the pitch angle for an indoors walk computed with a FOG IMU which we consider as a ground truth. The turquoise curve represents the pitch angle for the same walk processed with the pocket attitude estimator.

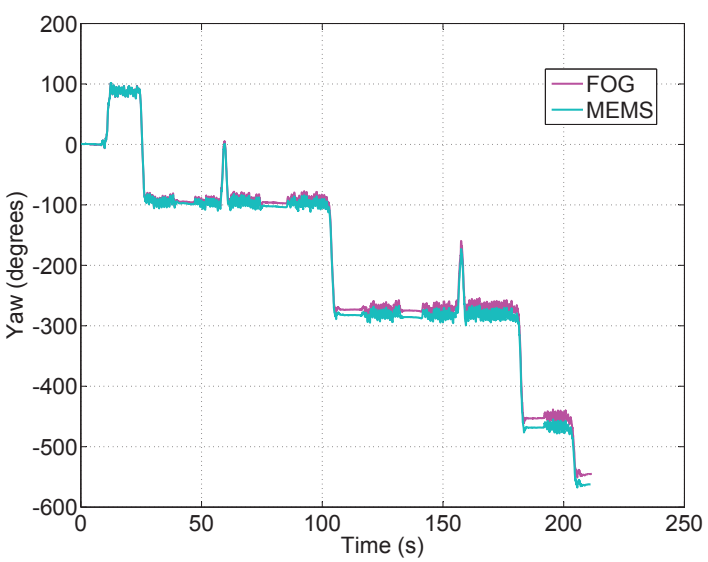

Fig. 15. The magenta curve represents the yaw angle for an indoors walk computed with a FOG IMU which we consider as a ground truth. The turquoise curve represents the yaw angle for the same walk processed with the pocket attitude estimator.

consider this is a good starting point, the improvement of the heading estimation is also under investigation because of its importance later on for the odometries, as previously explained.

1) Attitude Estimator for Handheld Positions: The described navigation system has been developed for pocket located IMUs. However, we are also interested in the rest of the handheld positions, i.e. swinging, calling and texting. It has been decided to test the attitude estimator also with the rest of handheld positions and these tests have been also done with the FOG IMU as a reference.

We have selected 3 different walks, which are represented in the Figure 16 for swinging, calling and texting respectively. For these 3 outdoor walks the volunteers were asked to describe a round trip path with a 180 degrees turn to go back to the initial position. Figure 16 represents the yaw angles, because the heading is a crucial variable for PDR systems. The magenta curves represent the ground truth extracted from 

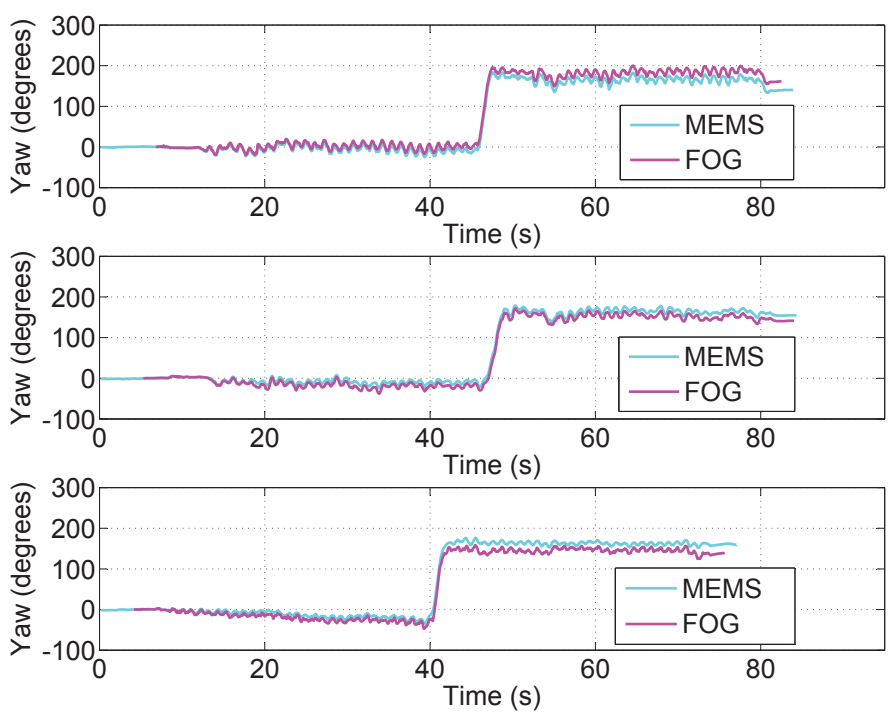

Fig. 16. These curves represent a round trip walk with a 180 degrees turn between the seconds $40-50$. The magenta curves represent the ground truth, because they have been taken from a FOG, while the cyan curves have been taken with a MEMS IMU and processed with our attitude estimator. For the first subplot the IMUs were held swinging, for the second one calling and for the third one texting.

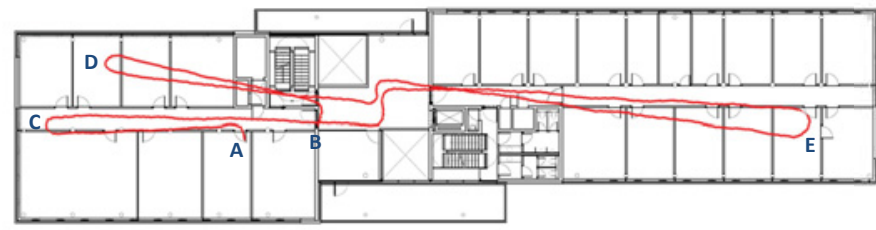

Fig. 17. The red curve represents the odometry of a 200 seconds walk in our office building. "A" is the starting point and "B" is the end of the walk. There is a displacement of 5 meters, because on the true trajectory this two points coincide.

the FOG IMU and the cyan curves represent the same walk processed with our pocket attitude estimator.

We consider these results satisfactory, also the roll and pitch estimation. As an outlook, the developed attitude estimator could be a solid starting point for the rest of handheld positions.

\section{B. Pocket Navigation System Experiments}

In order to assess the complete pocket navigation system, the odometries of the already described set of walks realized by 8 different volunteers have been processed. For the odometry, the floor plan is shown as a ground truth.

For clarity, we have decided to show the odometry of the walk described in the last subsection whose Euler angles roll, pitch and yaw can be seen in Figures 13, 14 and 15 in comparison with the ground truth.

In Figure 17, the red line represents their odometry. In this figure several errors can be observed. Firstly, the heading error introduces a 4 meters displacement because the point " $D$ " should be in the corridor. The starting point is labeled as "A" and the ending point is labeled as "B". For the true trajectory these two points are the same. The true trajectory describes the "C" and the "D" labels as the same point as well. Therefore, on the left part of the odometry, for the second time the user visits the corridor, there is a displacement of around 5 meters. Additionally, the "E" point should be at the end of the corridor, 3 meters further. This introduces a displacement of around 3 meters on the right part of the odometry. This source of errors can be divided in two and it is completely independent of inaccurate heading estimation. The displacement could be caused either by false step detection or by inaccurate step length estimation.

This result and the very similar odometries obtained for the complete set of walks realized by 8 volunteers, invite us to further investigate on new techniques for step detectors and step length estimators.

\section{CONCLUSIONS AND OUTLOOK}

Within this work we have created a standalone inertial pocket navigation system. We have started by developing a new attitude estimator. In contrast of the major part of the related work on PDR systems, apart of the heading, we estimate as well the angles roll and pitch. We have obtained satisfactory results for pocket orientations. Although it represents a good starting point for the heading estimation, due to the great influence of the yaw angle on the odometries, more techniques for correcting this angle should be further investigated.

We have tried our attitude estimator also for the rest of handheld positions, i.e. texting, swinging and phoning, and the results are optimistic. We are convinced this could be a solid starting point for a complete navigation system embedded in a smartphone.

For the develope of our magnetic disturbances detector, we have analyzed the magnetic field for different heights of the sensor. We have found the results quite interesting and we think the magnetic field should be studied as well in the third dimension. The related work shows a magnetic signature highly modulated indoors. This is optimal for slamming the floor, and we think we could apply this as well at the height of the pocket (and the rest of handheld positions). Moreover, it would be really interesting to study the change on the magnetic signature with the height for distinguishing the transition between the handheld positions.

With the odometries we have found a vast range of improvements that should be further investigated in order to obtain a better step detection and step length estimation.

\section{ACKNOWLEDGEMENTS}

The authors would like to thank Patrick Robertson for the interesting attitude conversations and Francisco Javier Fuentes Sánchez for his good ideas and support.

\section{REFERENCES}

[1] D. Titterton and J. Weston, Strapdown Inertial Navigation Technology, 2004.

[2] S. Rajagopal, "Personal Dead Reckogning System with Shoe Mounted Inertial Sensors," Ph.D. dissertation, 2008.

[3] E. Foxlin, "Pedestrian Tracking with Shoe-Mounted Inertial Sensors," IEEE Computer Graphics and Applications, pp. 38-46, December 2005. 
[4] B. Krach and P. Robertson, "Cascaded Estimation Architecture for Integration of Foot-Mounted Inertial Sensors," Location and Navigation Symposium IEEE/ION, 2008.

[5] A. Jimenez, F. Seco, J.Prieto, and J.Guevara, "A Comparison of Pedestrian Dead Reckoning Algorithms Using a Low-Cost MEMS IMU," IEEE International Symposium on Intelligent Sygnal Processing, pp. 37-42, August 2009.

[6] J. W. Kim, H. J. Jang, D.-H. Hwang, and C. Park, "A step, stride and heading determination for the pedestrian navigation system," Journal of Global Positioning Systems, vol. 3, no. 1-2, pp. 273-279, 2004.

[7] P. Goyal, V. J. Ribeiro, H. Saran, and A. Kumar, "Strap-down pedestrian dead-reckoning system," in Indoor Positioning and Indoor Navigation (IPIN), 2011 International Conference on. IEEE, 2011, pp. 1-7.

[8] M. H. Afzal, V. Renaudin, and G. Lachapelle, "Use of earth magnetic field for mitigating gyroscope errors regardless of magnetic perturbation," Sensors, vol. 11, no. 12, pp. 11390-11414, 2011.

[9] F. Zampella, M. Khider, P. Robertson, and A. Jiménez, "Unscented kalman filter and magnetic angular rate update (maru) for an improved pedestrian dead-reckoning," in Position Location and Navigation Symposium (PLANS), 2012 IEEE/ION. IEEE, 2012, pp. 129-139.

[10] A. Kim and M. Golnaraghi, "A quaternion-based orientation estimation algorithm using an inertial measurement unit," in Position Location and Navigation Symposium, 2004. PLANS 2004. IEEE, 2004, pp. 268-272.

[11] E. Kraft, "A quaternion-based unscented kalman filter for orientation tracking," in Proceedings of the Sixth International Conference of Information Fusion, vol. 1, 2003, pp. 47-54.

[12] M. Wang, Y. Yang, R. R. Hatch, and Y. Zhang, "Adaptive filter for a miniature mems based attitude and heading reference system," in Position Location and Navigation Symposium, 2004. PLANS 2004. IEEE, 2004, pp. 193-200.

[13] S. O. Madgwick, "An efficient orientation filter for inertial and inertial/magnetic sensor arrays," Report $x$-io and University of Bristol (UK), 2010.

[14] E. Foxlin, "Pedestrian tracking with shoe-mounted inertial sensors," Computer Graphics and Applications, IEEE, vol. 25, no. 6, pp. 38-46, 2005.

[15] A. Jimenez, F. Seco, C. Prieto, and J. Guevara, "A comparison of pedestrian dead-reckoning algorithms using a low-cost mems imu," in Intelligent Signal Processing, 2009. WISP 2009. IEEE International Symposium on. IEEE, 2009, pp. 37-42.

[16] W.-Y. Shih, L.-Y. Chen, and K.-C. Lan, "Estimating walking distance with a smart phone," in Parallel Architectures, Algorithms and Programming (PAAP), 2012 Fifth International Symposium on. IEEE, 2012, pp. $166-171$.

[17] C.-C. Lo, C.-P. Chiu, Y.-C. Tseng, S.-A. Chang, and L.-C. Kuo, "A walking velocity update technique for pedestrian dead-reckoning applications," in Personal Indoor and Mobile Radio Communications (PIMRC), 2011 IEEE 22nd International Symposium on. IEEE, 2011, pp. $1249-1253$.

[18] U. Steinhoff and B. Schiele, "Dead reckoning from the pocket-an experimental study," in Pervasive Computing and Communications (PerCom), 2010 IEEE International Conference on. IEEE, 2010, pp. 162-170.

[19] Y. Jin, H.-S. Toh, W.-S. Soh, and W.-C. Wong, "A robust dead-reckoning pedestrian tracking system with low cost sensors," in Pervasive Computing and Communications (PerCom), 2011 IEEE International Conference on. IEEE, 2011, pp. 222-230.

[20] S. Shin, M. Lee, C. Park, and H. S. Hong, "Pedestrian dead reckoning system with phone location awareness algorithm," in Position Location and Navigation Symposium (PLANS), 2010 IEEE/ION. IEEE, 2010, pp. 97-101.

[21] D. Gusenbauer, C. Isert, and J. Krosche, "Self-contained indoor positioning on off-the-shelf mobile devices," in Indoor Positioning and Indoor Navigation (IPIN), 2010 International Conference on. IEEE, 2010, pp. 1-9.

[22] M. Alzantot and M. Youssef, "Uptime: Ubiquitous pedestrian tracking using mobile phones," in Wireless Communications and Networking Conference (WCNC), 2012 IEEE. IEEE, 2012, pp. 3204-3209.

[23] D. Alvarez, R. C. González, A. López, and J. C. Alvarez, "Comparison of step length estimators from weareable accelerometer devices," in Engineering in Medicine and Biology Society, 2006. EMBS'06. 28th Annual International Conference of the IEEE. IEEE, 2006, pp. 5964 5967.

[24] J. Jahn, U. Batzer, J. Seitz, L. Patino-Studencka, and J. Gutiérrez Boronat, "Comparison and evaluation of acceleration based step length estimators for handheld devices," in Indoor Positioning and Indoor Navigation (IPIN), 2010 International Conference on. IEEE, 2010, pp. 1-6.

[25] Z. Yu, "Dead reckoning algorithms for indoor localization," Master's thesis, National University, Singapore, 2012

[26] V. Renaudin, V. Demeule, and M. Ortiz, "Adaptative pedestrian displacement estimation with a smartphone," internacional Conference on Indoor Positioning and Indoor Navigation, vol. 12, pp. 916-924, 2013

[27] S. Shin, C. Park, J. Kim, H. Hong, and J. Lee, "Adaptive step length estimation algorithm using low-cost mems inertial sensors," in Sensors Applications Symposium, 2007. SAS'07. IEEE. IEEE, 2007, pp. 1-5.

[28] V. Renaudin, M. Susi, and G. Lachapelle, "Step length estimation using handheld inertial sensors," Sensors, vol. 12, no. 7, pp. 8507-8525, 2012.

[29] S. Arulampalam, S. Maskell, N. Gordon, and T. Clapp, "A Tutorial on Particle Filters for On-line Non-linear/Non-Gaussian Bayesian Tracking," IEEE Transactions on Signal Processing, 2002.

[30] "Munich Earth Observatory. Monthly Magnetograms." http://www.geophysik.uni-muenchen.de/observatory/geomagnetism, 2014.

[31] V. Renaudin, M. Afzal, and G. Lachapelle, "Complete Triaxis Magnetometer Calibration in the Magnetic Domain," Journal of Sensors, 2010.

[32] M. Angermann, M. Frassl, B. Doniec, B. Julian, and P. Robertson, "Characterization of the Indoor Magnetic Field for Applications in Localization and Mapping," International conference on Indoor Positioning and Indoor Navigation (IPIN), November 2012.

[33] M. Afzal, V. Renaudin, and G. Lachapelle, "Assessment of Indoor Magnetic Field Anomalies Using Multiple Magnetometers," ION GNSS, Portland, Oregon, USA, 2010.

[34] B. Gozick, K. Subbu, R. Dantu, and T. Maeshiro, "Magnetic Maps for Indoor Navigation," IEEE Transactions on, vol. 60, no. 12, pp. 3883 3891, December 2011. 